\title{
Intervenção do profissional de educação física: formação, perfil e competências para atuar no Programa Academia da Saúde
}

Intervention of Physical Education professional: training, profile and skills to perform in the Programa Academia da Saúde in Brazil

Intervención del Profesional de Educación Física: formación, perfil y competencias para actuar en el Programa Academia de la Salud brasileño

Flávio Renato Barros da Guarda

Centro Acadêmico de Vitória, Universidade Federal de Pernambuco, Recife, Pernambuco, Brasil

Centro de Pesquisas Aggeu Magalhães, Fundação Oswaldo Cruz, Recife, Pernambuco, Brasil

Rafaela Niels da Silva

Programa de Pós-graduação Integrado em Saúde Coletiva,

Universidade Federal de Pernambuco, Recife, Pernambuco, Brasil

José Luiz do Amaral Correia de Araújo Júnior

Centro de Pesquisas Aggeu Magalhães, Fundação Oswaldo Cruz,

Recife, Pernambuco, Brasil

\author{
Maria Imaculada de Fátima Freitas \\ Escola de Enfermagem, Universidade Federal de Minas Gerais, \\ Belo Horizonte, Minas Gerais, Brasil \\ Pedro Miguel dos Santos Neto \\ Centro de Pesquisas Aggeu Magalhães, Fundação Oswaldo Cruz, \\ Recife, Pernambuco, Brasil
}

\section{RESUMO}

A inserção do profissional de Educação Física (PEF) no Sistema Único de Saúde (SUS) evidencia-se nas políticas e programas que possibilitam e demandam sua atuação. Nesse contexto, o presente estudo visou discutir aspectos relativos à formação, perfil e competências para a intervenção do PEF no âmbito do SUS, em especial no Programa Academia da Saúde (PAS), considerando a contribuição de diversos autores brasileiros quanto ao ensino ou à prática em Educação Física. Esta revisão da literatura analisou estudos produzidos no Brasil entre 1997 e 2013, investigando características de formação profissional, perfil e competências para atuação na área da saúde. A literatura revisada neste artigo apontou importantes avanços na formação em saúde, visando a integralidade e a superação do modelo biomédico. Entretanto, estudos realizados sobre a intervenção do PEF no SUS têm demonstrado divergências entre o perfil necessário e a formação no ensino superior. A atuação do PEF no SUS, e, especificamente no PAS, demanda o domínio de conteúdos teóricos, técnicas e vivências no campo da saúde coletiva, bem como a integração das instituições formadoras com os serviços de saúde, sobretudo por meio de canais permanentes de retroalimentação e discussão de problemas, avanços e necessidades.

Palavras-chave: Educação Física e Treinamento; Sistema Único de Saúde; Área de Atuação Profissional; Educação Superior; Recursos Humanos em Saúde; Pessoal de Saúde.

\section{INTRODUÇÃO}

A prática regular de atividades físicas vem sendo identificada como importante estratégia de promoção da saúde', pois promove uma série de benefícios biopsicossociais $2,3,4$, sendo considerada pelo Ministério da Saúde (MS) brasileiro como prioridade entre as

\footnotetext{
Correspondência / Correspondence / Correspondencia:

Flávio Renato Barros da Guarda

Rua Cassilândia, $n^{\circ}$ 331, bloco 1, apto. 304. Bairro: Várzea

CEP: 50740-370 Recife-Pernambuco-Brasil

Tel.: + 55 (81) 99984-1556

E-mail: flaviodaguarda@hotmail.com
}

estratégias da Política Nacional de Promoção da Saúde (PNPS), tanto na rede básica, quanto por meio de ações intersetoriais e de aconselhamento e divulgação'.

No Brasil, iniciativas vêm apresentando resultados promissores, como é o caso dos programas Agita São Paulo, Curitibativa e Academia da Cidade ${ }^{5}$. Nesse contexto, cabe ressaltar que a PNPS serviu de suporte político-institucional para a criação de mecanismos de apoio financeiro a programas de incentivo à prática de atividades físicas no âmbito do Sistema Único de Saúde (SUS), permitindo que estados e municípios pudessem implantar e implementar programas e projetos de promoção da saúde com foco no incentivo à prática de atividades físicas ${ }^{6}$. 
Entre 2006 e 2010, os municípios brasileiros que tiveram projetos submetidos e aprovados em portarias e editais do MS receberam recursos para a implantação ou implementação de estratégias de promoção da saúde em eixos prioritários da PNPS. Foram investidos mais de 125 milhões de reais em diversos municípios e no Distrito Federal, o que possibilitou a expansão dos programas de promoção da saúde e da atividade física ${ }^{6}$.

Tais iniciativas, entretanto, necessitavam de mecanismos de transferência regular de recursos e normatização, de modo a assegurar a sustentabilidade e melhorar o custo-efetividade. Diante dessa demanda e visando contribuir para a promoção da saúde por meio da implantação de polos com infraestrutura material e pessoal qualificado para a orientação de modos de vida saudáveis, práticas corporais, atividades físicas e de lazer, o MS instituiu, em 2011, o Programa Academia da Saúde (PAS) ${ }^{7}$, a ser implantado pelas secretarias de saúde do Distrito Federal e dos municípios, com suporte técnico das esferas estadual e federal do SUS.

PAS financia a construção de polos caracterizados como espaços públicos voltados à promoção da saúde e de atividades físicas ${ }^{8}$, e credita aos municípios e ao Distrito Federal desenvolver as ações sob coordenação da equipe da Atenção Primária em Saúde (APS), em articulação com toda a rede de serviços de saúde e outros equipamentos sociais, sob a normatização das políticas nacionais de promoção da saúde e da atenção básica ${ }^{8}$.

Nesse novo contexto, criou-se um importante campo de atuação para o profissional de Educação Física (PEF) que, ao se engajar nas atividades da APS, passa a enfrentar desafios relativos à articulação dos conhecimentos adquiridos na formação acadêmica e às (in)certezas, (ir)regularidades e improvisos da área da saúde, cujas peculiaridades deveriam ser constantemente apresentadas às instituições de ensino para que sejam consideradas na formação, perfil e competências profissionais, em processo constante de retroalimentação da formação/atuação.

Pressupõe-se, assim, que a relação entre formação e vida profissional exige interação entre ensino e serviço, para que sejam agregados novos conhecimentos à prática profissional e essa última interrogue, reforce ou modifique conteúdos, perfis e competências no ensino 9,10 .

Vale interrogar, portanto, sobre a incorporação da demanda atual do sistema de saúde no ensino da Educação Física, no que diz respeito à responsabilidade e competências exigidas para a intervenção do PEF em programas de promoção da atividade física, tais como o PAS.

Reflexões recentes acerca da educação profissional têm incluído questões como: i) que perfis profissionais os cursos de formação devem buscar; ii) que tipo de profissional estaria melhor preparado para oferecer serviços de qualidade e adequados às necessidades da sociedade; iii) que tipos de competências e sensibilidades devem ser esperadas de um profissional; iv) a relação entre autonomia, busca por conhecimento e motivação por parte dos profissionais; v) relação entre capacidade de resolver problemas e habilidade para aplicar soluções já estabelecidas; e vi) a relação entre a habilidade de realizar diagnósticos antes de prescrições e a capacidade de apenas fazer prescrições ${ }^{11}$.

Especificamente na Educação Física brasileira, - foco das pesquisas sobre a educação profissional encontra-se nos temas relativos à legislação ${ }^{12}$, organização e diretrizes curriculares ${ }^{13,14}$; e relação com o campo de conhecimento ${ }^{15}$. No entanto, a discussão sobre a organização do ensino e as demandas do setor saúde para atividades de PEF, nesse campo, ainda é muito recente e inconclusa.

Assim, este estudo visou discutir aspectos relacionados à formação, perfil e competências para a intervenção do PEF no âmbito do SUS, e as possíveis aplicações para a atuação no PAS, considerando a contribuição dos diferentes autores brasileiros nos âmbitos do ensino ou da prática em Educação Física, para compreender a pertinência do primeiro em relação ao segundo.

\section{MÉTODOS}

Realizou-se uma revisão da literatura produzida no Brasil acerca das características de formação, perfil e competências para atuação do PEF no setor saúde e, em especial, no PAS, no período de 1997 (ano em que a Educação Física foi reconhecida como profissão da saúde) a 2013 (ano da redefinição do PAS por parte do MS).

Procedeu-se uma busca por publicações oficiais dos Ministérios da Saúde e Educação brasileiros, além de pesquisa nos sítios eletrônicos da Biblioteca Virtual em Saúde e da Scientific Eletronic Library Online, utilizando-se os descritores: Educação Física e Treinamento; Desenvolvimento de pessoal; Competência profissional; Perfil profissional; e Pessoal de saúde; além de suas combinações. Os critérios de seleção foram: apresentar a contextualização da formação, perfil ou competência para atuação do PEF para atuar no campo da saúde; ter sido publicado no período de 1997 a 2013; e estar disponível na íntegra nos bancos de dados. Foram identificados 216 artigos, dos quais 53 foram incluídos.

\section{AS (IN)CERTEZAS DO CONHECIMENTO ACADÉMICO E SUA CONTRIBUIÇÃO PARA A FORMAÇÃO E A INTERVENÇÃO PROFISSIONAL}

Conhecimento assume um papel de destaque na formação e na atuação de diversas categorias profissionais. Importantes transformações sociais têm colocado a informação como principal recurso econômico da atualidade, diferentemente do que ocorria em períodos históricos anteriores ${ }^{16,17}$. Nesse contexto, a maioria das sociedades vem experimentando mudanças que dão origem ao que Drucker ${ }^{18}$ denomina de Sociedade do Conhecimento. $\bigcirc$ acesso a dados e informações torna-se cada vez mais fácil e rápido, 
caracterizando o conhecimento como uma estrutura em frequente transformação, e não mais estável e imutável como se apresentava no passado ${ }^{17}$.

Como consequência desse constante processo de transição, o mercado de trabalho é diretamente influenciado, pois a dinâmica na produção do conhecimento tem conduzido mudanças no modo de atuação de várias categorias profissionais, o que poderá acarretar alterações nas profissões já existentes, surgimento de novas e a extinção de outras nos próximos anos ${ }^{19}$.

Essas mudanças e as consequentes demandas profissionais produzem reflexos diretos sobre os processos de formação e aprendizagem que ocorrem para as diversas profissões, inclusive a Educação Física.

Para Freire et $\mathrm{al}^{20}$ :

[...] O graduando em Educação Física, futuro profissional, terá como responsabilidade a prestação de serviços à sociedade. Para isso, seu curso de graduação deverá compreender um saber profissional sobre sua área de intervenção, que lhe permita tomar as decisões mais adequadas em seu trabalho, capacitando-o para transformar o ambiente.

O saber profissional fundamenta-se na capacidade de mobilizar recursos cognitivos para o enfrentamento de diversas situações do cotidiano do trabalho, os quais compreendem dimensões conceituais, procedimentais e atitudinais inerentes à profissã $0^{21}$.

A primeira dimensão evidencia-se na capacidade de ajustar o conhecimento às particularidades do indivíduo e grupos, o que demanda o domínio de conceitos, fatos e princípios que formam o saber sobre o trabalho em Educação Física ${ }^{20}$. Logo, o profissional deve estar apto a compreender, entre outras coisas, o contexto social, político e cultural no qual será realizada sua intervenção ${ }^{22}$, as respostas fisiológicas do exercício, suas implicações sobre 0 desenvolvimento motor, condicionantes e determinantes sociais e ambientais da prática de atividades físicas, a predisposição do indivíduo para a prática e vários outros aspectos inerentes à atividade física. A dimensão conceitual contribui para que o profissional possa tomar as decisões corretas em sua intervençãa ${ }^{20}$.

Tais saberes, entretanto, não podem se limitar a aspectos biológicos da intervenção do $\mathrm{PEF}^{23}$. Sobretudo no campo da Saúde Coletiva, a formação profissional deve aproximar-se e incorporar questões sociais e os movimentos micropolíticos da vida ${ }^{9,24}$.

A atuação no campo da saúde, e mais especificamente no PAS, demanda do PEF o domínio de conhecimentos relativos às ciências sociais e humanas ${ }^{22}$, que envolvem a capacidade de identificar, descrever e problematizar questões sanitárias, na tentativa de compreendê-las e interpretá-las ${ }^{25}$.

É também importante verificar condicionantes e determinantes do processo saúde-doença e da prática da atividade física ${ }^{26}$ de cada indivíduo e da população, de modo a subsidiar a escolha de alternativas que melhor se adequem às necessidades de informação, orientação e práticas sobre o movimento humano.

Cabe, ainda, ao PEF identificar os objetivos e planejar as intervenções para implementação das ações mais adequadas a cada situação e público. Tais atividades compõem a dimensão procedimental inerente à profissão $0^{20,27}$.

O conhecimento relativo às dimensões conceitual e procedimental não garantem, por si só, uma intervenção eficiente, sendo necessária a observância e a busca pelo cumprimento de normas, valores e atitudes que configuram a terceira dimensão (dimensão atitudinal). Esta constitui o saber ser, e é operacionalizada por meio de condutas e valores que caracterizem uma ação ética ${ }^{13,20}$.

Essas dimensões do conhecimento fundamentam e devem guiar a preparação profissional, inclusive para a atuação no âmbito da saúde. Por outro lado, a qualidade da formação não pode limitar-se apenas a aprender a aprender, aprender a fazer, aprender a ser e aprender a conviver. Além do saber profissional, que caracteriza e delimita a intervenção, a formação deve estar diretamente relacionada ao papel social e político do trabalho $0^{9,23}$.

De acordo com Bosi ${ }^{28}$, o conhecimento formal é um elemento essencial, tanto para 0 reconhecimento social, quanto para a identidade de qualquer categoria profissional. Para a autora, a abrangência e a profundidade desse conhecimento estruturam o núcleo propriamente dito do saber de uma profissão. Dessa forma, o ensino superior reveste-se de fundamental importância, pois oferece a base cognitiva de uma profissão ${ }^{13,28,29}$.

Pode-se considerar que uma das características fundamentais de uma profissão é a existência de um saber profissional ou o conjunto de conhecimentos que é aprendido principalmente em cursos de graduação 28,29 .

No Brasil, esse conjunto de conhecimentos é ordenado por meio das diretrizes de formação específicas para cada curso de graduação, permitindo que as instituições de ensino de nível superior organizem seus currículos com base em critérios estabelecidos pelos Ministérios da Educação ${ }^{30}$ e Saúde ${ }^{31}$ sem, contudo, perder a flexibilidade e a autonomia para quaisquer adaptações ou adequações às realidades regionais e locais ${ }^{30}$.

Além das questões formais relacionadas ao conhecimento acadêmico, Soriano e Winterstein ${ }^{15}$ apontaram que a intervenção profissional fundamenta-se fortemente nas transformações derivadas de experiências, e não de explicações científicas. Para esses autores, o conhecimento baseado na prática profissional permite compreender em que momento e medida os conhecimentos científicos e acadêmicos participam do processo de construção da intervenção.

Ao questionar o que deve caracterizar um curso de formação de nível universitário e a necessidade de um curso superior para a formação profissional, 
Bagrichevsky e Estevão ${ }^{32}$ afirmaram a necessidade de uma formação mais complexa e elaborada, com maior aporte técnico-científico e nível de reflexão crítica, capaz de habilitar o indivíduo ao enfrentamento de dificuldades contextuais do cotidiano e adquirir esses mesmos conhecimentos, inclusive fora da universidade. Logo, (re)pensar finalidades, pertinências e principais problemáticas são práticas salutares de análise da legitimidade social de uma profissão ${ }^{32}$, sendo reflexão obrigatória nas instituições de formação.

Tal reflexão, entretanto, torna-se ainda mais difícil diante da complexidade e particularidades do setor saúde.

\section{(IR)REGULARIDADES DE UM SETOR COMPLEXO: O QUE É PRÓPRIO DA SAÚDE É PECULIAR À EDUCAÇÃO FÍSICA?}

A formação profissional em saúde apresenta características bastante específicas. $\bigcirc$ setor sanitário compõe o conjunto de atividades denominadas serviços de consumo coletivo e sofre as mesmas consequências do processo de ajuste macroestrutural que outros setores vêm sofrendo nos últimos anos, tais como a redução de custos, privatizações e terceirizações ${ }^{33}$.

No Brasil, a formação de recursos humanos para a saúde é diretamente influenciada pelo Movimento da Reforma Sanitária ${ }^{34}$, que inaugurou uma nova forma de se pensar e atuar em saúde, transformando os processos de formação profissional ${ }^{34,35}$. Nas últimas décadas, as instituições formadoras têm se esforçado para incorporar as reflexões a respeito dos modelos de atenção e propostas de intervenção baseadas no ato de cuidar, além de estratégias de ação potencialmente mais integrais e efetivas ${ }^{14,36}$.

Tais propostas, entretanto, não são suficientes para abarcar todas as necessidades de preparação profissional em saúde. Autores como Carvalho e Ceccim 9 , Akerman e Feverwerker ${ }^{37}$, e Anjos e Duarte ${ }^{34}$ apontaram que a formação de recursos humanos em saúde tem priorizado o caráter biológico, com centralização de conteúdos, limitando-se à transmissão de "informações" de forma enciclopédica, e se baseando, quase que exclusivamente, no binômio doença/reabilitação.

As Diretrizes Curriculares Nacionais ${ }^{30}$ sugerem formações de caráter generalista, humanista, crítico e reflexivo, numa tentativa de superação da perspectiva centrada no modelo hegemônico pela aproximação com novas tendências no cuidar, o que demanda a necessidade de currículos integrados e o uso de metodologias que privilegiem a participação ativa dos estudantes na construção do conhecimento e que contemplem "fatores de exposição"9. Essa construção conduz o estudante a uma melhor compreensão do processo saúde-doença e dos limites da ação uniprofissional no cuidar ${ }^{9,38}$.

Formar recursos humanos para a saúde implica na superação da visão biologicista. Consiste, segundo Vieira et $a^{33}$, em articular políticas sanitárias, de ciência e tecnologia, e de educação, de modo a atender às demandas do sistema de saúde, de acordo com as necessidades da população.

Sobre a formação de recursos humanos para a saúde, Anjos e Duarte ${ }^{34}$ afirmaram que:

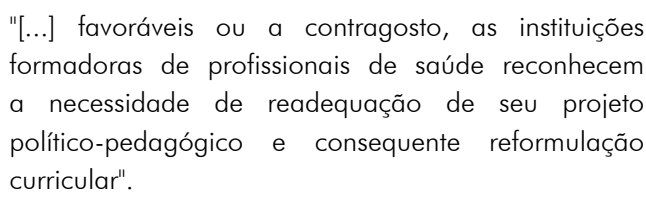
formadoras de profissionais de saúde reconhecem a necessidade de readequação de seu projeto político-pedagógico e consequente reformulação curricular".

Espera-se que o cuidado integral e a promoção da saúde estejam incluídos no projeto político-pedagógico, pois vêm sendo utilizados como principais ferramentas na tentativa de superação do modelo médico-hegemônico. Para a utilização de tais ferramentas, os serviços básicos de saúde têm ampliado suas equipes, incorporando outras categorias profissionais, como os de Educação Física ${ }^{34,39}$.

A integralidade é um eixo prioritário das políticas de saúde e pode ser analisada sob as dimensões da organização dos serviços, dos conhecimentos e das práticas dos trabalhadores do setor, e da formulação de políticas sanitárias ${ }^{40}$. Essas dimensões articulam-se às perspectivas da promoção da saúde, pois possibilitam a participação e habilitam outros atores, como usuários, gestores e demais colaboradores da sociedade à coparticipação e corresponsabilização pela sua saúde individual e coletiva ${ }^{41}$. Nesse sentido, a perspectiva de que a integralidade configura-se como o conjunto de ações e serviços em todos os níveis de complexidade, voltados à prevenção e ao cuidado individuais e coletivos ${ }^{42}$ e que a promoção da saúde é uma importante estratégia para garantir às pessoas condições de bem-estar físico, social e mental, demandam a atuação de profissionais capazes de intervir sobre aspectos físicos, educacionais e de inclusão social, como é o caso do PEF.

Embora formalmente incorporada às ações e aos serviços de saúde no SUS ${ }^{39,43}$, a Educação Física, como uma área de atuação profissional, encontra dificuldades de adequação da formação às necessidades e realidade do sistema público de saúde brasileiro ${ }^{22,44,45}$. Se, por um lado, o número de cursos, a oferta de vagas e a quantidade de egressos da graduação em Educação Física foi a segunda maior entre todos os cursos da área da saúde nos últimos anos ${ }^{32,46}$, por outro, as divergências quanto ao seu objeto e propostas de intervenção desencadearam a divisão da formação em bacharelado e licenciatura ${ }^{43,47}$, o que trouxe reflexos ainda não superados para a formação.

As Diretrizes Curriculares de $2004^{42}$ estabeleceram que a formação do graduado em Educação Física deve dotar os profissionais de habilidade específica para atuarem em diferentes campos que identifiquem expressões e manifestações da atividade física/motricidade humana, ou seja, nos diferentes contextos em que o movimento humano se manifesta, inclusive na saúde ${ }^{42}$. Entretanto, a formação em nível de graduação parece não atender às necessidades e especificidades do setor saúde ${ }^{34,48}$. 
Analisando a formação do PEF em instituições de ensino superior do Estado de São Paulo, Anjos e Duarte ${ }^{34}$ investigaram os objetivos dos cursos, as disciplinas relacionadas à saúde coletiva, e a existência de disciplinas de estágio em serviços de saúde. Como resultados, observaram que não há direcionamento dos cursos para o campo da saúde, embora os bacharéis tenham demonstrado maior respaldo em saúde do que os licenciados. $\bigcirc$ estudo identificou, ainda, o predomínio de disciplinas focadas em abordagens curativas e a ausência de um conjunto de disciplinas de Saúde Coletiva, sendo que algumas instituições nem as contemplavam. Os autores não identificaram nenhum curso que oferecesse estágio na Atenção Primária dos serviços públicos de saúde.

Em outra pesquisa, Souza e Loch $^{48}$ atribuíram parte da dificuldade de inserção e consolidação dos PEF no setor saúde ao direcionamento dos cursos de graduação, os quais, segundo os autores, têm caráter generalista para a área esportiva, em detrimento a uma pequena aproximação com a saúde coletiva.

Essas incertezas a respeito da formação e atuação, entretanto, não são exclusivas do campo da Educação Física. Alguns autores apontaram a necessidade de um melhor direcionamento da formação superior para o atendimento das necessidades, particularidades e complexidade do setor saúde $34,36,37$.

Akerman e Feverwerker ${ }^{37}$ afirmaram que a abordagem do processo saúde-doença focada exclusivamente no referencial biologicista é insuficiente para qualquer intervenção em saúde e, portanto, qualquer formação restrita a esse referencial será deficitária. Tal afirmação parece corroborar Carvalho e Ceccim 9 ao apontarem que a discussão sobre a formação em saúde demanda tematizar o ensino no âmbito das graduações.

Para Paim e Almeida Filho ${ }^{36}$, ensino e pesquisa em saúde devem direcionar-se ao serviço básico e à educação em saúde, dentro de uma visão ampla que considere as pessoas, a comunidade, a família e as relações sociais. Vários autores ${ }^{9,34,36,48}$ apontaram os conhecimentos sobre saúde coletiva como fundamentais para o exercício profissional, instrumentalizando a compreensão sobre a organização, o funcionamento dos serviços de saúde, as características e peculiaridades dos modelos de atenção, a epidemiologia e vigilância em saúde das comunidades, devendo ser ofertados nos cursos de graduação na área de saúde.

No contexto da saúde coletiva, a atuação do PEF no PAS configura-se como importante desafio para a consecução dos princípios do SUS, sobretudo o da integralidade, o qual fundamenta as ações da Atenção Primária. Esse programa está incluído nas ações do primeiro nível de atenção à saúde desde 2011, sob a responsabilidade dos municípios e do Distrito Federal, tendo como objetivo principal: "contribuir para a promoção da saúde e produção do cuidado e de modos de vida saudáveis da população a partir da implantação de polos com infraestrutura e profissionais qualificados ${ }^{149}$.
Esses "profissionais qualificados"49, incluem os que atuam na Atenção Primária (Equipes de Saúde da Família e dos Núcleos de Apoio à Saúde da Família - NASF), bem como trabalhadores de outras áreas do conhecimento para a execução de atividades específicas aos objetivos, princípios e diretrizes do Programa. Cabe ressaltar, que a atuação do PEF torna-se fundamental para o cumprimento do objetivo específico número 10 (aumentar o nível de atividade física da população) e desenvolvimento das atividades relativas ao eixo 1 (práticas corporais e atividades físicas) previstos na portaria que redefiniu o PAS em $2013^{49}$.

A intervenção do PEF nos polos do PAS implica, necessariamente, em mobilizar e selecionar conhecimentos inerentes à Educação Física, bem como articular tais saberes aos desenvolvidos e apresentados pelos demais atores do processo de cuidado (profissionais, usuários, gestores, e demais colaboradores), na perspectiva do trabalho em equipes multiprofissionais. Esse desafio vem sendo descrito como inerente a todas as categorias profissionais da saúde ${ }^{9,36}$, e transcende a esfera do conhecimento científico e da formação acadêmica ${ }^{15}$.

Por outro lado, as demais profissões da saúde parecem estar mais ambientadas com a rotina e alguns conceitos do campo da saúde, enquanto a formação acadêmica do PEF ainda carece incluí-lo para instrumentalizar adequadamente os egressos e profissionais para lidar com questões como: intersetorialidade, humanização, referência, contrarreferência, integralidade, clínica ampliada e apoio matricial. Esses são conceitos pouco utilizados nos cursos de graduação e pós-graduação da área ${ }^{50}$, o que pode dificultar a intervenção desses trabalhadores junto ao setor sanitário.

\section{PERFIL PROFISSIONAL}

Para Saviani ${ }^{51}$, a relação entre educação e trabalho nem sempre foi linear. Com o surgimento da propriedade privada na antiguidade, a educação inicialmente atendia a indivíduos abastados e com sustento garantido, prestando-se mais à formação de lideranças políticas e militares do que à preparação para a inserção no sistema produtivo. Com o surgimento das cidades modernas, atribui-se à escola a função de formar cidadãos cientes de seus direitos e deveres. Ademais, os avanços ocorridos na economia, nas ciências e na tecnologia também contribuíram para que os trabalhadores escolarizados fossem reconhecidos como mais habilitados a lidar com a complexidade crescente do sistema produtivo ${ }^{52}$.

Para Fogaça ${ }^{53}$, educação e formação profissional passaram a se integrar, principalmente por meio de dois processos: i) a globalização, definida como o conjunto de mudanças que instituíram novas formas de relação entre os países nos campos econômico, social, cultural, político e tecnológico; ii) o surgimento e crescimento de um complexo produtivo baseado na automação flexível. Nessa perspectiva, os três níveis de ensino (fundamental, médio e superior) passam a integrar 
as discussões da agenda da reestruturação produtiva e, consequentemente, das suas inter-relações com o mercado de trabalho ${ }^{54}$, demandando maior articulação entre instituições formadoras, empresas e comunidade ${ }^{55}$.

Tais mudanças, segundo Gondim ${ }^{52}$, têm conduzido as instituições formais a se reestruturarem e isso se reflete no delineamento de um perfil profissional mais compatível com a nova realidade. As novas demandas criadas a partir do fenômeno da globalização exigem profissionais com competências diversas e habilidades para lidar com várias situações. Esse perfil profissional baseia-se em três grandes grupos de habilidades $^{52}$ : a) cognitivas; b) técnicas especializadas; e c) comportamentais e atitudinais.

a) Habilidades cognitivas - normalmente relacionadas à criatividade, conhecimento geral, julgamento crítico, raciocínio lógico e abstrato, e solução de problemas. São obtidas principalmente pelos processos formais de educação;

b) Técnicas especializadas - relacionam-se com o domínio de uma habilidade, conhecimentos de informática, língua estrangeira, operação de equipamentos e processos de trabalho; e

c) Habilidades comportamentais e atitudinais têm relação com a predisposição do indivíduo para a cooperação, responsabilidade, motivação, busca constante por aperfeiçoamento (atitude de aprender a aprender), iniciativa e ética.

Assim, o perfil configura-se como referência que permite às instituições de ensino estabelecer nexos entre teoria e prática no processo de formação superior ${ }^{52}$, e aos graduandos conhecer o campo de trabalho e avaliar os caminhos efetivos de emprego, comparando de forma racional as habilidades que dispõem com as demandas do mercado ${ }^{56}$ em todas as áreas profissionais, inclusive no setor saúde.

Características específicas do trabalho em saúde demandam profissionais que sejam capazes de planejar, organizar, desenvolver e avaliar ações que respondam às necessidades de indivíduos e comunidades, na articulação com os diversos setores envolvidos na promoção da saúde ${ }^{57}$. Nesse sentido, aspectos relacionados ao indivíduo e à formação dos trabalhadores que atuam no setor saúde podem influenciar tanto na escolha da profissão e de área de atuação, quanto no trato com questões inerentes à execução do trabalho ${ }^{58}$. $\bigcirc$ conhecimento do perfil desses trabalhadores pode revelar questões referentes à sua identificação com o trabalho e à preparação para a atuação nos serviços de saúde ${ }^{57}$, além de contribuir para o aperfeiçoamento, tanto dos serviços quanto das instituições formadoras. Para Schiappacasse et $\left.a\right|^{59}$, a base para a organização do processo de formação, bem como a avaliação do currículo, demandam a determinação desse perfil. Logo, torna-se fundamental que as instituições formadoras desenvolvam e reestruturem permanentemente os perfis das carreiras profissionais sob sua responsabilidade.
A proposta do PAS demanda que o trabalho se desenvolva na perspectiva da promoção e da intervenção sobre condicionantes e determinantes sociais do processo saúde-doença ${ }^{49}$, exigindo que os perfis de diferentes categorias profissionais se alinhem às características e necessidades do trabalho interdisciplinar. No caso específico da Educação Física, a formação de nível superior parece não ter preparado os profissionais para 0 atendimento dos preceitos constitucionais do SUS $34,48,60,61$.

Um estudo desenvolvido por Fonseca et al60 apontou algumas causas para esse desencontro, destacando-se, entre outras: i) divergências entre - perfil necessário para atuar no setor saúde e a formação de docentes para o ensino superior; e ii) incompatibilidade do perfil desejado para o bacharel em Educação Física (tido como muito abrangente e pouco consistente) e o atendimento ao eixo da integralidade no cuidado.

Esses autores ainda destacaram a necessidade de repensar a preparação profissional para além da reserva de mercado, direcionando-a para a formação de um perfil que se identifique com o conceito ampliado de saúde, com habilidade para implementar ações coletivas e para estimular o empoderamento da população.

A autonomia para tomar decisões, solucionar problemas relativos ao trabalho em saúde e empoderar a população requer a renovação da formação e da prática, o que pode ser conseguido com o desenvolvimento de competências profissionais.

\section{COMPETÊNCIAS ESPERADAS POR MEIO DA FORMAÇÃO PROFISSIONAL}

A renovação das práticas pode ser alcançada pelo estímulo ao desenvolvimento de competências, o que, segundo Faustino ${ }^{62}$, figura como uma nova perspectiva para a formação dos profissionais de saúde, incentivando a reflexão crítica e viabilizando respostas às novas demandas sociais e do trabalho, favorecendo, inclusive, o desenvolvimento da cidadania.

conceito de competência tem vários sentidos, podendo ser definida como: a capacidade de utilizar habilidades, atitudes e conhecimentos; destreza para utilizar o conhecimento, visando atingir um determinado objetivo; habilidade para mobilizar conhecimentos adquiridos para a prática profissional; e competência para articular saberes - saber fazer, saber ser, saber agir ${ }^{63}$.

Segundo Ramos ${ }^{64}$, competência é "[...] mecanismo subjacente que permite a integração de múltiplos conhecimentos e atos necessários à realização da ação", sendo condição de desempenho, pois expressa os recursos articulados e mobilizados frente a uma situação. Nesse sentido, o desempenho seria produto das competências, e várias competências podem ser requeridas para a realização de apenas uma ação ${ }^{64}$. Para a Organização Internacional 
do Trabalho (OIT), os conceitos de competência profissional e de formação e qualificação profissional baseados em competências surgiram nos Estados Unidos na década de $1960^{65}$. Com a crise estrutural do capitalismo no final dos anos 1980, a discussão sobre o modelo de competências chegou ao meio empresarial, demandando ações do setor educação, em função das exigências do sistema produtivo ${ }^{66}$. Isso se evidenciou na educação a partir da Lei de Diretrizes e Bases e das reformas educacionais orientadas e financiadas por organismos internacionais, tais como - Banco Interamericano de Desenvolvimento, a OIT, a Organização das Nações Unidas para a Educação, a Ciência e a Cultura, entre outros, denotando o atendimento às necessidades de articular e subordinar as ações do setor educação às necessidades do setor produtivo ${ }^{66,67}$.

A competência caracteriza-se pela elaboração de planos e arranjos que permitem mobilizar os conhecimentos, no momento oportuno e com discernimento, sendo a capacidade de orquestração destes arranjos. O que caracteriza a competência de um especialista é a capacidade de dominar rapidamente situações comuns com arranjos que entram em ação automaticamente. Além disso, o especialista coordena e diferencia rapidamente estratégias de ação e conhecimentos de modo a enfrentar situações inéditas ${ }^{64}$.

O desenvolvimento de competências apresenta-se, assim, como uma nova perspectiva para a formação dos profissionais de saúde, tanto por estimular uma análise mais crítica das situações vivenciadas no ambiente de trabalho e no processo saúde-doença, quanto por possibilitar respostas às demandas sociais, contribuindo para o desenvolvimento da cidadania ${ }^{62,67}$.

As perspectivas de mudança do modelo de atenção em saúde, com vistas à priorização de ações de promoção e prevenção, em detrimento das curativas', bem como as diretrizes, características e demandas específicas das APS, requerem do PEF competências para compreender a organização (inclusive política) e o funcionamento dos sistemas e serviços de saúde, (re)ver conceitos sobre o processo saúde-doença e trabalhar nas perspectivas da interdisciplinaridade e da intersetorialidade ${ }^{50}$.

Importantes estudos sobre competências do PEF em diversos campos de atuação como escolas, academias de ginástica e na área dos esportes têm descrito que essas se relacionam basicamente às dimensões de conhecimentos, habilidades e atitudes $50,68,69,70$.

Para a intervenção no PAS, no qual as ações serão desenvolvidas em parceria com as equipes de Saúde da Família, NASF e outros atores locais e regionais? faz-se necessário que o PEF seja capaz de dominar saberes e técnicas inerentes à atuação no campo da saúde e da educação, articulando-as às experiências e peculiaridades das pessoas e da comunidade.
A noção de vínculo como ferramenta capaz de ampliar as ações dos profissionais de saúde e permitir a atuação na perspectiva da educação em saúde, discussões e atividades de grupo $^{71}$, representa um importante instrumento de atuação do PEF no PAS. O estabelecimento de vínculos com a população pode caracterizar a construção de competências para o $\mathrm{PEF}$, pois congrega aspectos teóricos como território e humanização; habilidades inerentes ao campo da atividade física e da saúde coletiva, como a escuta qualificada e os comportamentos necessários ao convívio e articulação de ações com gestores, usuários e outras categorias profissionais ${ }^{72}$.

O trabalho junto às equipes dos NASF demanda, ainda, do PEF, a competência para lidar com questões específicas do trabalho na Atenção Primária como apoio matricial e clínica ampliada.

O apoio matricial é um arranjo organizacional e uma metodologia de gestão do trabalho em saúde que visa ampliar as possibilidades de ação da Equipe de Saúde da Família e do trabalho multiprofissional ${ }^{73}$.

A clínica ampliada é a superação da clínica tradicional, fragmentada e focada na doença. Baseia-se na compreensão ampliada do processo saúde-doença, na construção compartilhada de diagnósticos e terapêuticas, na ampliação do objeto de trabalho, na transformação dos meios ou instrumentos de trabalho e no suporte aos profissionais de saúde ${ }^{74}$. Busca considerar enfermidade, sujeito e contexto como focos para o estudo, o conhecimento e a intervenção, além do permitir ao PEF qualificar sua intervenção, pois exige, além do conhecimento, uma postura positiva e problematizadora do seu trabalho e da interação deste com o processo de trabalho das demais categorias profissionais ${ }^{72}$.

\section{CONCLUSÃO}

A inserção do PEF no SUS evidencia-se nas políticas e programas que possibilitam e demandam sua atuação, consolidando-se a partir da incorporação às equipes dos NASF e da possibilidade de atuação no PAS.

Entretanto, a atuação desses trabalhadores enfrenta as (in)certezas, (ir)regularidades e improvisos de um setor complexo e de um campo de atuação ainda pouco explorado e retroalimentado pelo próprio sistema de saúde e pelas instituições formadoras, pois a relação entre educação profissional e intervenção demanda interação mútua e articulação entre ensino, serviços e vivências práticas.

A literatura revisada neste artigo apontou importantes avanços na formação em saúde, visando a integralidade e a superação do modelo biomédico. Estudos na área da formação em Educação Física têm contemplado questões relativas à normatização do ensino e às diretrizes curriculares, sobretudo no que se refere à atuação na escola, entretanto, as 
investigações sobre a organização do ensino para atender às demandas sociais geradas pela inserção dessa categoria no setor público da saúde ainda são incipientes. Nessas, evidenciou-se a necessidade de aproximação da formação às demandas do trabalho em saúde, sobretudo em função da ausência de conteúdos, disciplinas, estágios e outras vivências no campo da saúde coletiva.

descompasso entre formação acadêmica e demandas do mercado de trabalho é observado em todas as profissões da saúde, inclusive na Educação Física, caracterizando-se pela fragmentação do conhecimento, priorização do caráter biológico e reprodução de práticas prescritivas focadas na doença, protocolos e procedimentos.

$\bigcirc$ processo de reestruturação produtiva, caracterizado pela desregulamentação e flexibilização do trabalho a partir da década de 1970, conduziu a importantes transformações na preparação profissional, levando as instituições formadoras ao delineamento de perfis profissionais compatíveis com a realidade de um mundo globalizado, inclusive no setor saúde. Essas novas demandas requerem trabalhadores dotados de habilidades cognitivas, técnicas e comportamentais específicas para sua área de atuação, além da capacidade de articular conhecimentos e práticas para trabalhar em equipe. $\bigcirc$ perfil profissional torna-se, portanto, a base para a organização do processo de formação e avaliação curricular. Entretanto, os estudos realizados sobre a intervenção do PEF no SUS têm demonstrado divergências entre o perfil necessário e a formação dos docentes do ensino superior, além da incompatibilidade do perfil desejado para o bacharel ao atendimento às demandas do SUS.
Além do perfil, a competência profissional figura como importante componente para a intervenção do PEF nos serviços de saúde, caracterizando-se como a capacidade de organizar e utilizar diferentes recursos (cognitivos, técnicos e comportamentais) para a resolução de problemas relacionados ao trabalho. Esses recursos são discutidos na produção científica da Educação Física, a qual apresenta alguns importantes estudos sobre competências do PEF para atuar na escola, em academias de ginástica e na área esportiva, porém ainda existe uma importante lacuna no que se refere à investigação das competências do PEF para atuar no setor público da saúde.

Evidenciou-se, dessa forma, que tanto formação quanto perfil e competências demandam a aquisição, organização, articulação e aplicação de conhecimentos relacionados a conceitos, procedimentos e atitudes que conformam os saberes específicos de uma profissão. Esses não se constroem, nem se consolidam apenas com reformas curriculares ou reedição de diretrizes, pois estas variam historicamente em função de diferentes contextos socioeconômicos e políticos, e em atendimento às necessidades da população e do mercado de trabalho.

Nesse sentido, a atuação do PEF no SUS e especificamente no PAS demandam o domínio de conteúdos teóricos, técnicas e vivências no campo da saúde coletiva, bem como a integração das instituições formadoras com os serviços de saúde, sobretudo por meio de canais permanentes de retroalimentação e discussão de problemas, avanços e necessidades. Isso poderá contribuir para o diálogo entre os atores envolvidos (inclusive alunos), para a construção coletiva, a corresponsabilização e o estímulo à criticidade em defesa da prestação de serviços de qualidade para a população.

\section{Intervention of Physical Education professional: training, profile and skills to perform in the Programa Academia da Saúde in Brazil}

\section{ABSTRACT}

The insertion of Physical Education professional (PEP) in the health system (Sistema Único de Saúde - SUS) is evident in policies and programs that enable and require his/her performance. In this context, this study aims to discuss aspects of student's education, profile and skills in order to interfere the PEP under SUS, particularly in the community health and fitness program (Programa Academia da Saúde - PAS), considering the contribution of several Brazilian authors about teaching or practicing in Physical Education. This literature review analyzed studies that had been produced in the Country between 1997 and 2013, investigating education, profile and skills of professionals that will work in the health area. The literature review also mentioned important advances in health education aiming the integrality and overcoming of the biomedical model. However, studies on the intervention of the PEP at SUS have shown differences between the required profile and training in higher education. The performance of the PEP at SUS, and specifically on the PAS, requires the knowledge of theoretical concepts, techniques and experiences in the field of public health, as well as integrating educational institutions with health services especially by permanent channels of feedback and discussion of problems, progress and needs.

Keywords: Physical Education and Training; Unified Health System; Professional Practice Location; Education, Higher; Health Manpower; Health Personnel. 


\section{Intervención del Profesional de Educación Física: formación, perfil y competencias para actuar en el Programa Academia de la Salud brasileño}

\section{RESUMEN}

La inserción del profesional de Educación Física (PEF) en el Sistema Único de Salud (SUS) se evidencia en las políticas y programas que posibilitan y demandan su actuación. En ese contexto, el presente estudio tiene como objetivo discutir aspectos relativos a la formación, perfil y competencias para la intervención del PEF en el ámbito del SUS, en especial en el Programa Academia de la Salud (PAS), considerando la contribución de diversos autores brasileños con relación a la enseñanza o la práctica en Educación Física. Esta revisión de la literatura analizó estudios producidos en el Brasil entre 1997 y 2013, investigando características de formación profesional, perfil y competencias para actuar en el sector de la salud. La literatura revisada en este artículo señala importantes avances en la formación en salud para la integralidad y la superación del modelo biomédico. Sin embargo, estudios realizados sobre la intervención del PEF en el SUS, han demostrado divergencias entre el perfil necesario y la formación en la enseñanza superior. La actuación del PEF en el SUS, y, específicamente en el PAS demanda el dominio de contenidos teóricos, técnicas y vivencias en el campo de la salud colectiva, bien como la integración de las instituciones formadoras con los servicios de salud, sobre todo a través de canales permanentes de retroalimentación y discusión de problemas, avances y necesidades.

Palabras clave: Educación y Entrenamiento Físico; Sistema Único de Salud; Ubicación de la Práctica Profesional; Educación Superior; Recursos Humanos en Salud; Personal de Salud.

\section{REFERÊNCIAS}

1 Ministério da Saúde (BR). Secretaria de Vigilância em Saúde. Secretaria de Atenção à Saúde. Política Nacional de Promoção da Saúde. Brasília: Ministério da Saúde; 2006. (Série pactos pela saúde; 7).

2 Shephard BC, Stephens RJ, Sutton JR, McPherson $\mathrm{BD}$, editors. Exercise, fitness and health. Champaign: Human Kinetics; 1990. Exercise, fitness and health: the consensus statement; p. 3-28.

3 Pate RR, Pratt M, Blair SN, Haskell WL, Macera CA, Bouchard $C$, et al. Physical activity and public health: a recommendation from the Centers for Disease Control and Prevention and the American College of Sports Medicine. JAMA. 1995 Feb;273(5):402-7.

4 Ministério da Saúde (BR). Secretaria de Vigilância em Saúde. Departamento de Análise de Situação de Saúde. A vigilância, o controle e a prevenção das doenças crônicas não-transmissíveis: DCNT no contexto do Sistema Único de Saúde brasileiro. Brasília: Organização Pan-Americana da Saúde; 2005.

5 Hallal PC, Tenório MC, Tassitano RM, Reis RS, Carvalho YM, Cruz DK, et al. Avaliação do programa de promoção da atividade física Academia da Cidade de Recife, Pernambuco, Brasil: percepções de usuários e não-usuários. Cad Saude Publica. 2010 jan;26(1):70-8.

6 Malta DC, Castro AM, Cruz DKA, Gosh CS. A promoção da saúde e da atividade física no Sistema Único de Saúde. Rev Bras Atividade Fisica Saude. 2008;13(1):24-7.
7 Brasil. Ministério da Saúde. Portaria n ${ }^{\circ} 719$, de 7 de abril de 2011. Institui o Programa Academia da Saúde no âmbito do Sistema Único de Saúde. Diário Oficial da União, Brasília, p. 52, 8 abr. 2011 . Seção 1.

8 Brasil. Ministério da Saúde. Portaria n 1.401, de 15 de junho de 2011 . Institui, no âmbito da Política Nacional de Atenção Básica, o incentivo para construção de pólos da Academia da Saúde. Diário Oficial da União, Brasília, p. 107, 27 jun. 2011 . Seção 1.

9 Carvalho YM, Ceccim RB. Formação e educação em saúde: aprendizados com a saúde coletiva. In: Campos GWS, Minayo MCS, Akerman M, Drumond Júnior $M$, Carvalho YM, organizadores. Tratado de saúde coletiva. São Paulo: Hucitec; 2006. p. 149-82.

10 Mello ALSF, Moysés ST, Moysés SJ. A universidade promotora de saúde e as mudanças na formação profissional. Interface. 2010 jul-set;14(34):683-92.

11 Tani G. Avaliação das condições do ensino de graduação em Educação Física: garantia de uma formação de qualidade. Rev Mackenzie Educ Fis Esporte. 2007;6(2):55-70.

12 Souza Neto S, Alegre AN, Hunger D, Pereira JM. A formação do profissional de educação física no Brasil: uma história sob a perspectiva da legislação federal no século XX. Rev Bras Cienc Esporte. 2004 jan;25(2): 113-28.

13 Tani G. Professional preparation in physical education: changing labor market and competence. Motriz Rev Educ Fis. 2013 Jul-Sep;19(3):552-7. 
14 Braid LMC, Machado MFAS, Aranha AC. Estado da arte das pesquisas sobre currículo em cursos de formação de profissionais da área da saúde: um levantamento a partir de artigos publicados entre 2005 e 2011. Interface. 2012 jul-set; 16(42):679-92.

15 Soriano JB, Winterstein PJ. A constituição da intervenção profissional em educação física: interações entre o conhecimento "formalizado" e as estratégias de ação. Rev Bras Educ Fis Esporte. 2004 out-dez;18(4):315-32.

16 Machado NJ. Cidadania e educação. 4. ed. São Paulo: Escrituras; 1997. O futuro do trabalho e a educação; p. 9-28. (Coleção Ensaios Transversais).

17 Fartes VLB. Formação profissional, profissões e crise das identidades na sociedade do conhecimento. Cad Pesqui. 2008 set-dez;38(135):583-5.

18 Drucker P. Sociedade pós-capitalista. São Paulo: Pioneira; 1993.

19 Schwartz G. As profissões do futuro. São Paulo: Publifolha; 2000. Aperte os cintos, o mercado sumiu!

20 Freire ES, Verenguer RCG, Reis MCC. Educação física: pensando a profissão e a preparação profissional. Rev Mackenzie Educ Fis Esporte. 2002; $1(1): 39-46$.

21 Zabala A. Aprendizaje significativo: el profesor como movilizador de las competencias de sus alumnos. In: Anais do $6^{\circ}$ Seminário de Educação e Sociedade; 1997; São Paulo. São Paulo: Grupo Associação de Escolas Particulares; 1997. p. 1-39.

22 Carvalho YM. Saúde, sociedade e vida um olhar da educação física. Rev Bras Cienc Esporte. 2006 mai;27(3): 153-68.

23 Pina LD. Atividade física e saúde: uma experiência pedagógica orientada pela pedagogia histórico crítica. Motrivivencia. 2008 jun;20(30):158-68.

24 Merhy EE, Magalhães Júnior HM, Rimoli J, Franco TB, Bueno WS, organizadores. $\bigcirc$ trabalho em saúde: olhando e experenciando o SUS no cotidiano. São Paulo: Hucitec; 2003.

25 Nunes ED. As ciências humanas e a saúde: algumas considerações. Rev Bras Educ Med. 2003 jan-abr;27(1):65-71.

26 Seabra AFT, Mendonça DM, Thomis MA, Anjos LA, Maia JA. Determinantes biológicos e sócio-culturais associados à prática de atividade física de adolescentes. Cad Saude Publica. 2008 abr;24(4):721-36.

27 Coll C, Pozo JI, Sarabia B, Valls E. Os conteúdos na reforma: ensino e aprendizagem de conceitos, procedimentos e atitudes. 2. ed. Porto Alegre, RS: Artmed; 2000. A aprendizagem e o ensino dos procedimentos; p. 73-118.
28 Bosi MLM. Profissionalização e conhecimento: a nutrição em questão. São Paulo: Hucitec; 1996.

29 Freidson E. Renascimento do profissionalismo: teoria, profecia e política. São Paulo: Edusp; 1998.

30 Brasil. Ministério da Educação. Conselho Nacional de Educação. Câmara de Educação Superior. Resolução CNE/CES $N^{\circ} 4$, de 7 de novembro de 2001. Institui diretrizes curriculares nacionais do curso de graduação em medicina [Internet]; 2001 nov 7 [citado 2009 jan 20]. Disponível em: http:// portal.mec.gov.br/cne/arquivos/pdf/CES04.pdf.

31 Brasil. Lei $n^{\circ} 8.080$ de 19 de setembro de 1990. Dispõe sobre as condições para a promoção, proteção e recuperação da saúde, a organização e o funcionamento dos serviços correspondentes e dá outras providências. Diário Oficial da União, Brasília, 20 set. 1990. Seção 1.

32 Bagrichevsky M, Estevão A. Perspectivas para a formação profissional em educação física: o SUS como horizonte de atuação. Arq Mov. 2008 jan-jun;4(1): 128-43.

33 Vieira AS, Amâncio Filho A, Oliveira SP, Garcia ACP. Panorama das graduações em saúde no Brasil. In: Ministério da Saúde (BR). Fundação Oswaldo Cruz. Dinâmica das graduações em saúde no Brasil: subsídios para uma política de recursos humanos. Brasília: Ministério da Saúde; 2006. p. 25-37.

34 Anjos TC, Duarte ACGO. A educação física e a estratégia de saúde da família: formação e atuação profissional. Physis. 2009;19(4):1127-44.

35 Rocha VM, Centurião $\mathrm{CH}$. Profissionais da saúde: formação, competência e responsabilidade social. In: Fraga $A B$, Wachs $F$, organizadores. Educação física e saúde coletiva: políticas de formação e perspectivas de intervenção. Porto Alegre: Universidade Federal do Rio Grande do Sul; 2007. p. 17-31

36 Paim JS, Almeida Filho N. Saúde coletiva: uma "nova saúde pública" ou campo aberto a novos paradigmas? Rev Saude Publica. 1998 ago;32(4):299-316.

37 Akerman M, Feuerwerker L. Estou me formando (ou me formei) e quero trabalhar: que oportunidades o Sistema de Saúde me oferece na Saúde Coletiva? Onde posso atuar e que competências preciso desenvolver? In: Campos GWS, Minayo MCS, Akerman M, Drumond Júnior M, Carvalho YM, organizadores. Tratado de saúde coletiva. São Paulo: Hucitec; 2006. p. 183-98.

38 Ribeiro EC. Entre a emancipação e a regulação: limites e possibilidades da avaliação das escolas médicas [tese]. Rio de Janeiro: Universidade do Estado do Rio de Janeiro; 2001. 
39 Brasil. Ministério da Saúde. Portaria $n^{\circ} 154$, de 24 de janeiro de 2008. Cria os Núcleos de Apoio à Saúde da Família - NASF. Diário Oficial da União, Brasília, p. 47, 25 jan. 2008. Seção 1.

40 BVS Integralidade. $\bigcirc$ que é integralidade em saúde [Internet]? Rio de Janeiro: Fiocruz, Universidade do Estado do Rio de Janeiro; 2007 [citado 2014 jan 20]. Disponível em: http://www.bvsintegralidade. icict.fiocruz.br/php/level.php?lang = pt\&component $=$ $19 \&$ item $=9$.

41 Buss PM. Promoção da saúde e qualidade de vida. Cad Saude Coletiva. 2000 jan-mar;5(1):163-77.

42 Brasil. Ministério da Educação. Conselho Nacional de Educação. Parecer CNE/CES nº 058/2004. Estabelece as diretrizes curriculares nacionais para os cursos de graduação em educação física. Brasília: Ministério da Educação; 2004.

43 Brasil. Conselho Federal de Educação. Parecer $\mathrm{n}^{\circ}$ 215, de 11 de março de 1987. Dispõe sobre a reestruturação dos cursos de graduação em Educação Física, sua nova caracterização, mínimos de duração e conteúdo. Brasília: Conselho Federal de Educação; 1987.

44 Pasquim HM. A saúde coletiva nos cursos de graduação em educação física. Saude Soc. 2010 jan-mar; 19(1): 193-200.

45 Falci DM, Belisario SA. A inserção do profissional de educação física na atenção primária à saúde e os desafios em sua formação. Interface [Internet]. 2013 out-dez [citado 2014 fev 1];17(47):885-99. Disponível em: http://www. scielo.br/scielo.php?script=sci_arttext\&pid =S1414$32832013000400010 \&$ lng $=$ en\&nrm $=$ iso.

46 Garcia ACP, Amâncio Filho A, Vieira AS, Oliveira SP, Oliveira ES. Educação física. In: Ministério da Saúde (BR). Fundação Oswaldo Cruz. Dinâmica das graduações em saúde no Brasil: subsídios para uma política de recursos humanos. Brasília: Ministério da Saúde; 2006. p. 235-43.

47 Brugnerotto F, Simoes R. Caracterização dos currículos de formação profissional em Educação Física: um enfoque sobre saúde. Physis. 2009;19(1):149-72.

48 Souza SC, Loch MR. Intervenção do profissional de educação física nos Núcleos de Apoio à Saúde da Família em municípios do norte do Paraná. Rev Bras Ativ Fis Saude. $2011 ; 16(1): 5-10$.

49 Brasil. Ministério da Saúde. Portaria n 2.681, de 7 de novembro de 2013. Redefine o Programa Academia da Saúde no âmbito do Sistema Único de Saúde (SUS). Saúde Legis [Internet]; 2013 [citado 2014 fev 1]. Disponível em: http://bvsms.saude.gov.br/bvs/ saudelegis/gm/2013/prt2681_07_11_2013.html.

50 Coutinho SS. Competências do profissional de educação física na atenção básica à saúde [tese]. Ribeirão Preto: Universidade de São Paulo, Escola de Enfermagem de Ribeirão Preto; 2011.
51 Saviani D. O trabalho como princípio educativo frente às novas tecnologias. In: Ferretti CJ, Zibas DML, Madeira FR, Franco MLPB, organizadores. Novas tecnologias, trabalho e educação: um debate multidisciplinar. 2. ed. Petrópolis: Vozes; 1994. p. 151-68.

52 Gondim SMG. Perfil profissional e mercado de trabalho: relação com a formação acadêmica pela perspectiva de estudantes universitários. Estud Psicol. 2002 jul-dez;7(2):299-309.

53 Fogaça A, organizador. Políticas de emprego no Brasil. Campinas: Instituto de Economia Unicamp; 1998. A educação e reestruturação produtiva; p. 30-45.

54 Leite EM. Reestruturação produtiva, trabalho e qualificação no Brasil. In: Bruno L, organizador. Educação e trabalho no capitalismo contemporâneo: leituras selecionadas. São Paulo: Atlas; 1996. p. 146-87.

55 Dowbor L. Educação, tecnologia e desenvolvimento. In: Bruno L, organizador. Educação e trabalho no capitalismo contemporâneo: leituras selecionadas. São Paulo: Atlas; 1996. p. 17-40.

56 Werbel JD. Relationships among career exploration, job search intensity and job search effectiveness in graduating college students. J Vocat Behav. 2000 Dec;57(3):379-94.

57 Cotta RMM, Schott M, Azeredo CM, Franceschini SCC, Priore SE, Dias G. Organização do trabalho e perfil dos profissionais do Programa Saúde da Família: um desafio na reestruturação da atenção básica em saúde. Epidemiol Serv Saude. 2006 set; 15(3):7-18.

58 Guarda FRB, Silva RN, Tavares RAW. Perfil sociodemográfico dos médicos que compõem equipes de saúde da família na Região Metropolitana do Recife, Estado de Pernambuco, Brasil. Rev Pan-Amaz Saude. 2012 jun;3(2): 17-24.

59 Schiappacasse E, Ramírez L, Retamal F, Pérez H, Ibáñez P. Perfil profesional del médico. Educ Med Salud. 1984;18(4):359-70.

60 Fonseca AS, Menezes AS, Loch MR, Feitosa WMN, Nahas MV, Nascimento JV. Pela criação da Associação Brasileira de Ensino da Educação Física para a Saúde: Abenefs. Rev Bras Ativ Fis Saude. $2011 ; 16(4): 283-8$.

61 Rodrigues JD, Ferreira DKS, Silva PA, Caminha IO, Farias Júnior JC. Inserção e atuação do profissional de educação física na atenção básica à saúde: revisão sistemática. Rev Bras Ativ Fis Saude. 2013 jan; 18(1):5-15.

62 Faustino RLH. Saberes e competências na formação da enfermeira em saúde coletiva [tese]. São Paulo: Universidade de São Paulo, Escola de Enfermagem; 2003. 198 p. 
63 Mota LB, Aguiar AC. Novas competências profissionais em saúde e o envelhecimento populacional brasileiro: integralidade, interdisciplinaridade e intersetorialidade. Cienc Saude Coletiva. 2007 mar-abr;12(2):363-72.

64 Ramos MN. Qualificação, competências e certificação: visão educacional. Formação. 2001 mai;2:17-26.

65 Vargas F, Steffen I, Brígido R. Certificação de competências profissionais: análise qualitativa do trabalho, avaliação e certificação de competências: referenciais metodológicos. Brasília: Organização Internacional do Trabalho; 2002.

66 Deluiz N. O modelo das competências profissionais no mundo do trabalho e na educação: implicações para o currículo. Bol Tec Senac [Internet]. 2001 set-dez [citado 2014 fev 4];27(3):187-93. Disponível em: http://www.senac.br/informativo/ BTS/273/boltec273b.htm.

67 Marques CMS, Egry EY. As competências profissionais em saúde e as políticas ministeriais. Rev Esc Enferm Usp. 2011 mar;45(1):187-93.

68 Batista PMF, Matos ZMRP, Mesquita IMR, Graça ABS. Representações dos profissionais do desporto acerca do conceito de competência profissional. Rev Bras Educ Fis Esporte. 2011 abrjun;25(2): 197-213.
69 Feitosa WM. As competências específicas do profissional de educação física: um estudo Delphi [dissertação]. Florianópolis: Universidade Federal de Santa Catarina; 2002.

70 Nascimento JVA. As competências específicas do profissional de educação física e desportos: um estudo Delphi. Rev Horizonte. 1999;15(87):1-12.

71 Campos GWS. Saúde paidéia. São Paulo: Hucitec; 2003.

72 Freitas FF, Carvalho YM, Mendes VM. Educação física e saúde: aproximações com a "clínica ampliada". Rev Bras Cienc Esporte. 2013 jul-set;35(3):639-56.

73 Campos GWS, Domitti AC. Apoio matricial e equipe de referência: uma metodologia para gestão do trabalho interdisciplinar em saúde. Cad Saude Publica. 2007 fev;23(2):399-407.

74 Ministério da Saúde (BR). Secretaria de atenção à saúde. Política Nacional de Humanização da Atenção e Gestão do SUS. Clínica ampliada e compartilhada. Brasília: Ministério da saúde; 2009. 64 p. (Série B. Textos básicos de saúde).

Recebido em / Received / Recibido en: 14/6/2014 Aceito em / Accepted / Aceito en: 2/10/2014 